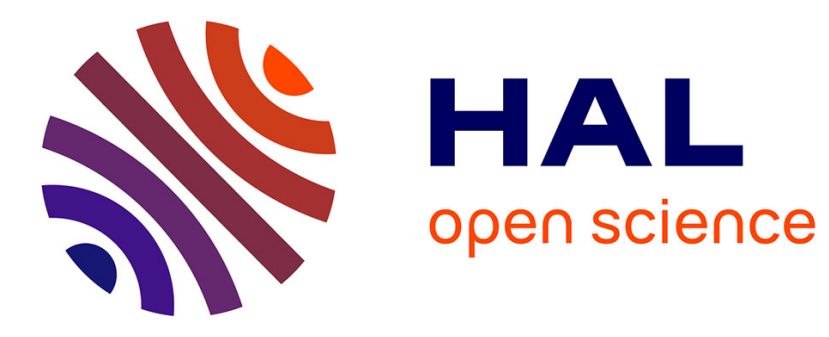

\title{
La fabrication du bleu égyptien à Cumes
}

\author{
Laetitia Cavassa, Paolo Caputo
}

\section{To cite this version:}

Laetitia Cavassa, Paolo Caputo. La fabrication du bleu égyptien à Cumes. Jean-Pierre Brun. L'artisanat antique en Italie méridionale. Mélanges offerts à Maria-Francesca Buonaiuto, 32, Centre Jean Bérard, pp.169-179, 2009. halshs-03090910

\section{HAL Id: halshs-03090910 \\ https://shs.hal.science/halshs-03090910}

Submitted on 1 Feb 2021

HAL is a multi-disciplinary open access archive for the deposit and dissemination of scientific research documents, whether they are published or not. The documents may come from teaching and research institutions in France or abroad, or from public or private research centers.
L'archive ouverte pluridisciplinaire HAL, est destinée au dépôt et à la diffusion de documents scientifiques de niveau recherche, publiés ou non, émanant des établissements d'enseignement et de recherche français ou étrangers, des laboratoires publics ou privés. 


\section{Artisanats antiQues}

D'Italie et DE GaUle MÉlanges OFFerts À Maria Francesca Buonaiuto
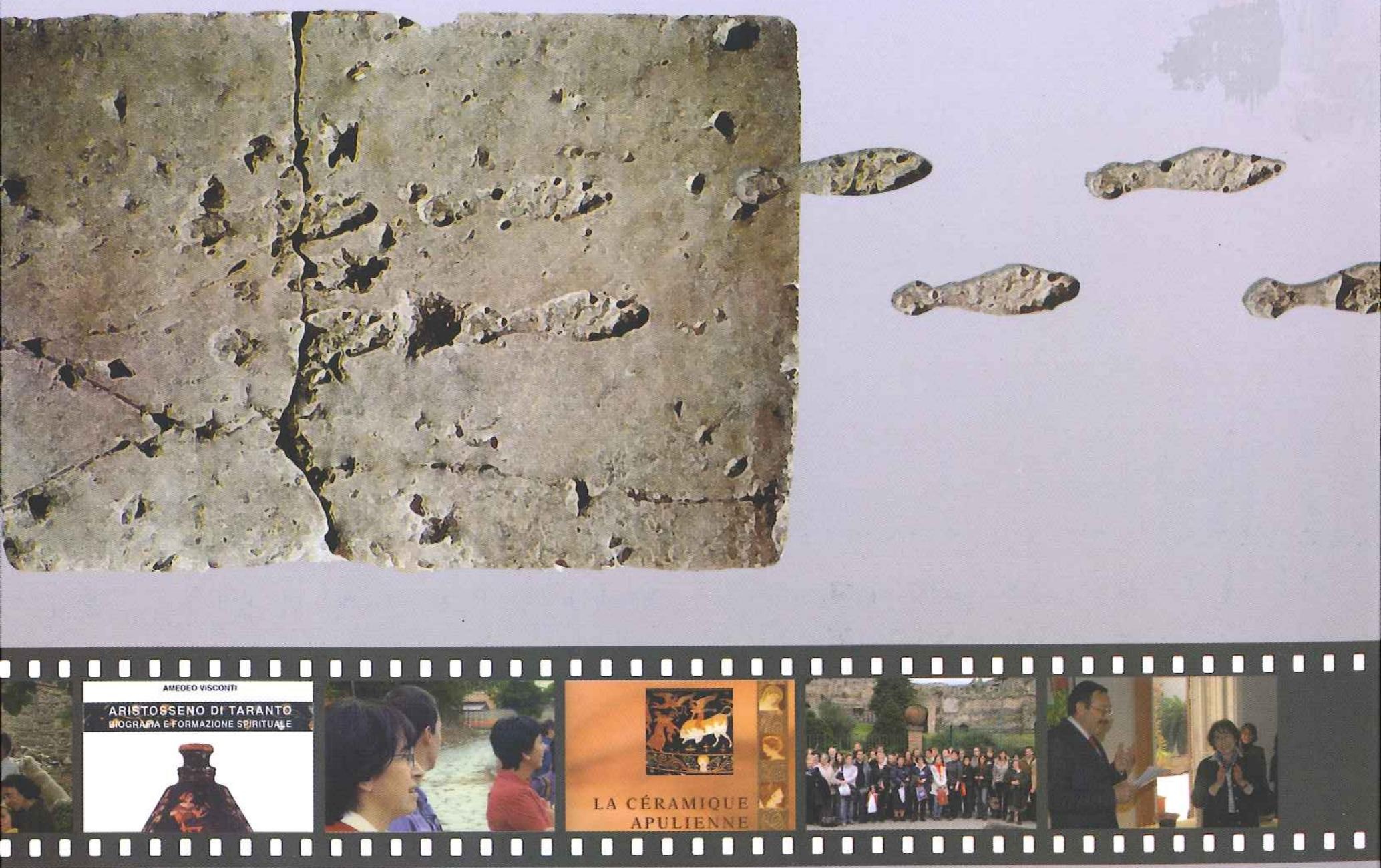

CNRS 
Collection du Centre Jean Bérard, 32

Archéologie de l'artisanat antique, 2

\section{Artisanats antiques d'Italie et de Gaule}

\section{Mélanges offerts à Maria Francesca Buonaiuto}

édités par Jean-Pierre Brun

Avec le soutien de

l'Agence Nationale de Recherche (programme Artifex) et du

Ministère des Affaires ÉtrangèRes et EuropéEnnes

Naples 2009 


\title{
La fabrication du bleu égyptien à Cumes
}

\author{
Paolo Caputo et Laëtitia Cavassa*
}

\section{Le premier pigment de synthèse}

Le bleu égyptien, qui porte ausi les noms de «bleu d'Alexandrie», «bleu Vestorien», «bleu de Pouzzoles », "bleu Pompéien", caeruleum ou encore hsbd iryt en égyptien hiéroglyphique, terme qui signifie littéralement «lapis-lazuli fabriqué», trouve son origine en Égypte.

Cette couleur est le premier pigment de synthèse créé par les Égyptiens durant la IV dynastie (26132494 avant J.-C. $)^{1}$ pour pallier la carence en minéraux naturels tels que le lapis-lazuli, pierre semiprécieuse, que sa rareté réservait à la bijouterie. Le pigment bleu sert aussi bien pour la fabrication d'objets, que pour la peinture, la mosaïque et l'écriture. Aujourd'hui, l'appellation «bleu égyptien » est le nom générique d'un silicate double de cuivre et de calcium dont la composition chimique est $\mathrm{CaCuSi}_{4} \mathrm{O}_{10}$.

\section{Le caeruleum à l'époque romaine}

Le bleu artificiel connut un grand succès en Égypte et plus tard dans le monde hellénistique et romain. À l'état de produit brut, il se présente sous la forme de petites boules de quelques centimètres de diamètre et c'est ainsi qu'on le trouve généralement dans les gisements archéologiques. Ce pigment fut rebaptisé par les auteurs latins, caeruleum Vestorianum, du nom de Vestorius, citoyen romain de Pouzzoles, "affairiste ${ }^{2}$ et ami de Cicéron que les auteurs tels que Vitruve et Pline l'Ancien présentent comme la personne ayant importé la recette du bleu égyptien d'Alexandrie à Pouzzoles.
Nous disposons de deux sources principales sur ce pigment. La première est le texte de Vitruve, écrit vers 27 avant notre ère. "La fabrication du bleu céruléen a été mise au point à Alexandrie, et plus tard Vestorius en a fondé une fabrique à Pouzzoles. C'est un produit tout à fait étonnant par les ingrédients à partir desquels il a été mis au point. On broie en effet du sable avec de la fleur de nitre, assez finement pour obtenir une sorte de farine ; et, lorsqu'on y mélange du cuivre à l'état de limaille à l'aide de grosses limes, on arrose le tout, pour qu'il s'agglomère ; puis en le roulant dans ses mains, on en fait des boulettes que l'on rassemble pour les faire sécher ; une fois sèches, on les met dans un pot de terre cuite, et les pots sont portés dans des fours : ainsi, quand le cuivre et le sable entrant en effervescence sous la violence du feu se sont fondus ensemble, en se donnant l'un à l'autre et en recevant l'un de l'autre leurs sueurs ils abandonnent leurs caractères individuels, et, leur être propre anéanti par la violence du feu, ils sont réduits à l'état de couleur bleu $»^{3}$.

Vitruve fournit donc trois informations majeures. La première est le nom de Vestorius, identifié comme étant le fabricant du caeruleum. J.-P. Morel a souligné l'importance de cette mention en ces termes : « ce qui, en fait, est extraordinaire, voire peut-

* P. Caputo : Funzionario responsabile dell'Ufficio per i Beni Archeologici di Cuma, Soprintendenza speciale per i Beni Archeologici di Napoli e Pompei.

L. Cavassa : Ingénieur CNRS, Centre Jean Bérard (USR 3133, CNRS-École française de Rome).

1 Forbes 1965, p. 224 ; Lee 2000, p. 109.

2 Andreau 1983, p. 18.

3 Vitruve, De l'architecture, VII, 11,1, traduction de Bernard Liou et Michel Zuinghedau (1995). 
être unique, c'est que Vitruve et Pline citent le nom d'un producteur de biens artisanaux $»^{4}$. Il s'agit à notre connaissance de la seule identification directe d'un artisan qu'on pourrait qualifier d'industriel en ce sens qu'il ne participait pas lui-même à la chaîne de production.

La deuxième information est la recette du pigment : "sable, fleur de nitre et cuivre ». Toutefois, cette recette reste incomplète. Il manque quelques informations nécessaires pour obtenir un bleu égyptien de bonne qualité : les quantités de chaque ingrédient, le temps de cuisson nécessaire et la température indispensable à la transformation du mélange en couleur bleue. Peut-on y voir un oubli volontaire de la part de l'artisan? Ne pas dévoiler la recette dans sa totalité pour garder le contrôle intégral sur la fabrication ? ${ }^{5}$

Enfin, Vitruve témoigne qu'au $I^{\text {er }}$ siècle avant notre ère, Pouzzoles abritait un ou plusieurs ateliers de bleu égyptien. Ceci est d'autant plus significatif que Pouzzoles, durant la période concernée, est le principal port de Rome et accueille les marchandises en provenance d'Orient.

Son texte pose toutefois un problème à propos de la mise au point du procédé à Alexandrie. S'agissant d'un produit inventé durant la $\mathrm{IV}^{\mathrm{e}}$ dynastie, il est impossible que la technique soit originaire d'Alexandrie, fondée en 323 avant notre ère par Alexandre le Grand. Mais c'est certainement à Alexandrie que les Romains ont eu connaissance de la technique dont ils ont tout naturellement crédité l'invention à la ville. De même que les marchandises voyagaient d'Alexandrie vers l'Italie, via Puteoli, les technologies nouvelles venant d'Orient transitaient également vers l'Occident.

La deuxième source est Pline l'Ancien. "L'azur est un sable. Il y en avait jadis trois espèces : l'égyptien, le plus apprécié ; le scythique, qui se délaye facilement et qui, une fois broyé, prend quatre couleurs, une claire et une foncée, une plus épaisse et une plus fine ; à l'azur scythique on préfère encore maintenant celui de Chypre. À ces variétés s'est ajouté l'azur de Pouzzoles et celui d'Espagne, quand on eut commencé à traiter le sable en ces endroits. Toute espèce d'azur passe à la teinture : on le fait bouillir avec une herbe qui lui convient et il en boit le suc. Pour le reste, la préparation est identique à celle de la chrysocolle. Avec de l'azur, on produit ce qu'on appelle le lomentum, (le «bleu à laver »); on l'obtient en lavant l'azur et en le broyant. Le « bleu à laver » est plus clair que l'azur; son prix est de 10 deniers la livre, celui de l'azur de 8 deniers. On l'emploi sur la craie, car il ne supporte pas la chaux. Le lomentum vestorien est une variété récente, qui tire son nom de celui de son inventeur ; on le produit avec la partie la plus fine de l'azur égyptien; son prix est de 11 deniers la livre. On fait le même usage de l'azur de Pouzzoles, et il s'emploie de plus pour les fenêtres ; on l'appelle cylon. On a commencé naguère à importer également de l'azur indien, dont le prix est de 7 deniers. En peinture, il s'emploie pour les contours, c'est-à-dire pour séparer les ombres de la lumière. Il existe encore une espèce de lomentum pilé ; on l'évalue à cinq as la livre. La pureté de l'azur se reconnaît à l'essai, s'il s'enflamme sur un charbon ardent ; on le falsifie en faisant bouillir dans de l'eau des violettes séchées et en en pressant le jus à travers un linge sur de la terre d'Érétrie. En médecine, l'azur a la propriété de nettoyer les plaies, ce qui fait qu'on l'incorpore aux emplâtres ainsi qu'aux cautères. Mais il se broie très difficilement. Dans ses usages médicinaux, il est légèrement mordant et astringent, et il cicatrise les plaies. On le brûle dans des récipients de terre pour le rendre efficace.

Nous n'ignorons pas que les prix que nous avons indiqués à l'occasion varient selon les endroits et changent presque tous les ans, suivant les coûts de la navigation, ou d'après les achats des particuliers, ou encore si un acquéreur en position dominante accapare la récolte de l'année : nous n'oublions pas le cas de Démétrius, accusé devant les consuls, sous le principat de Néron, par toute la corporation des parfumeurs de Capoue. Mais il nous a fallu indiquer les prix habituellement pratiqués à Rome, afin de donner une idée de la valeur normale des choses ${ }^{6}$.

Pline l'Ancien mentionne le lomentum vestorianum, produit de qualité selon ses dires, car « on le produit avec la partie la plus fine de l'azur égyptien ${ }^{7}$. Nous constatons donc qu'au $I^{\text {er }}$ siècle de notre ère, le bleu égyptien est toujours associé au nom de Vestorius et ceci alors que ce dernier est certainement mort depuis près d'un siècle ${ }^{8}$. La survivance du nom de Vestorius attaché à la production du caeruleum plus d'un siècle après en fait-il déjà un

\footnotetext{
4 Morel 1983, p. 29-30.

5 Nous renvoyons pour les détails concernant la recette de Vitruve à un article de F. Delamare (2003).

6 Pline l'Acien, Hist. Nat., XXXIII, 161-164, traduction de Hubert Zehnacker (1983).

7 Fit ex Aegyptii leuissima parte.

8 Nous ne possédons aucune information concernant sa vie après la mort de Cicéron qui mentionne Vestorius dans 23 lettres datées de 56 à 44 avant notre ère. V. Sirago a proposé de dater sa mort des années 20 avant notre ère (Sirago 1979).
} 


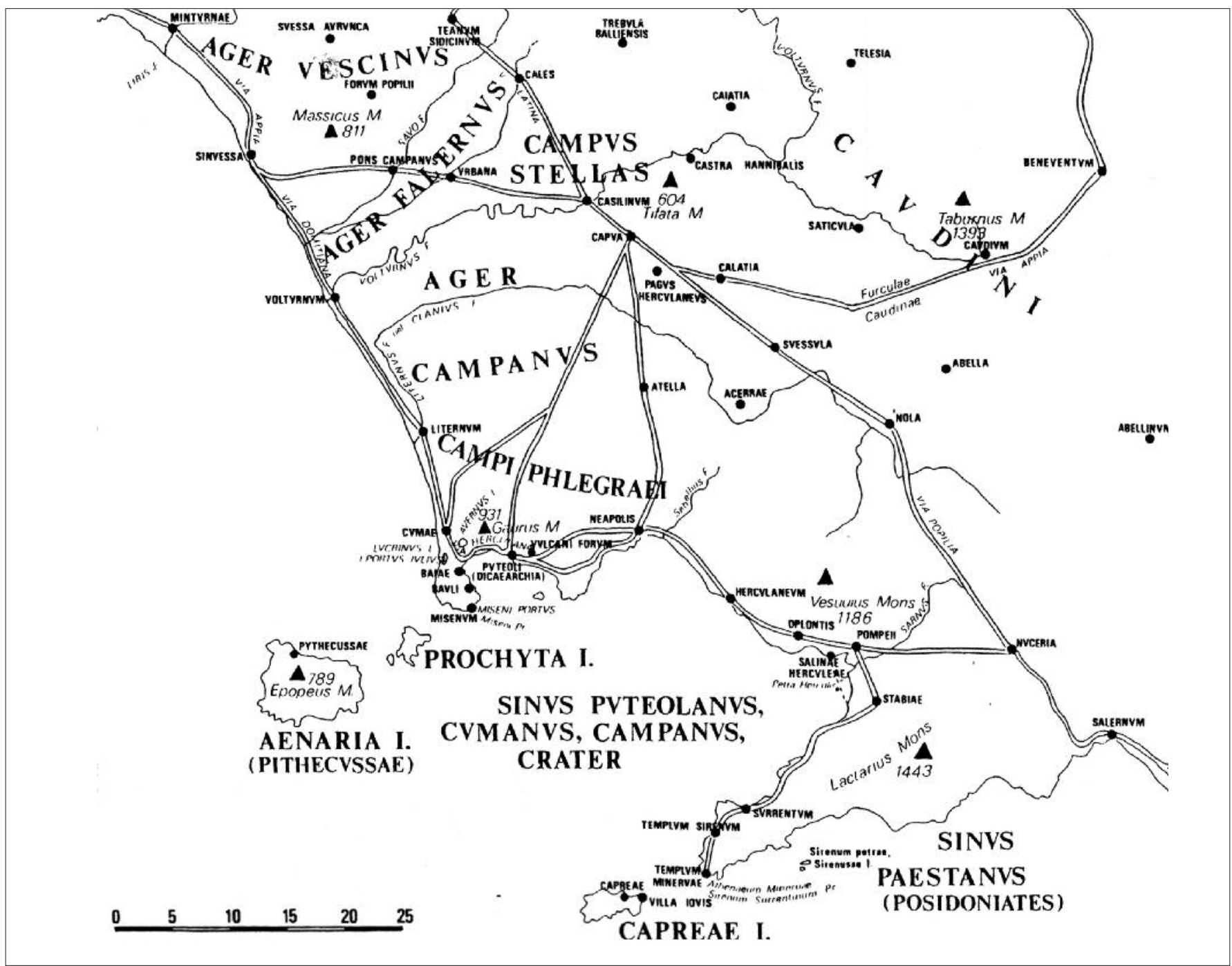

Fig. 1. Carte de la Campanie et des Champs Phlégréens (d'après Martin 1985, pl. I)

" label de qualité »? Rappelons qu'au début du IV ${ }^{\text {e }}$ siècle de notre ère, l'Édit du Maximum fixe le prix du cyaninum Vestorianum de 80 à 150 deniers la livre ${ }^{9}$. La mention du pigment associé au nom de la gens Vestoria confirme qu'à cette époque le produit était toujours associé au nom de son "inventeur» italien, même si le monopole de sa production n'appartenait certainement plus à cette famille. Le nom de «bleu Vestorien " était devenu un label de qualité, un nom commun et non plus un produit fabriqué à Pouzzoles par un membre de la gens Vestoria.

Pline précise que la production du pigment à Pouzzoles et en Espagne est liée aux débuts de l'exploitation du sable dans ces deux lieux. Ceci est un élément qui prend son importance pour la cité de Cumes, car Pline dit que le meilleur sable est celui du Volturne : « [...] à présent on utilise aussi un sable blanc venant du fleuve Volturne en Italie et que l'on trouve entre Cumes et Literne, sur six mille pas du rivage côtier, là où il est le plus tendre, et on le broie au mortier ou à la meule ${ }^{10}$. Cette information est intéressante car le sable est l'un des ingrédients essentiels à la composition du pigment. Le site de Cumes se trouve donc à proximité d'un gisement de sable de premier choix pour l'industrie du bleu égyptien.

\footnotetext{
9 Giacchero 1974, p. 218, n³4, 84 et 85.

10 Pline l'Ancien, Hist. Nat., XXXVI, 194, traduction de J. André et R. Bloch (1981).
} 


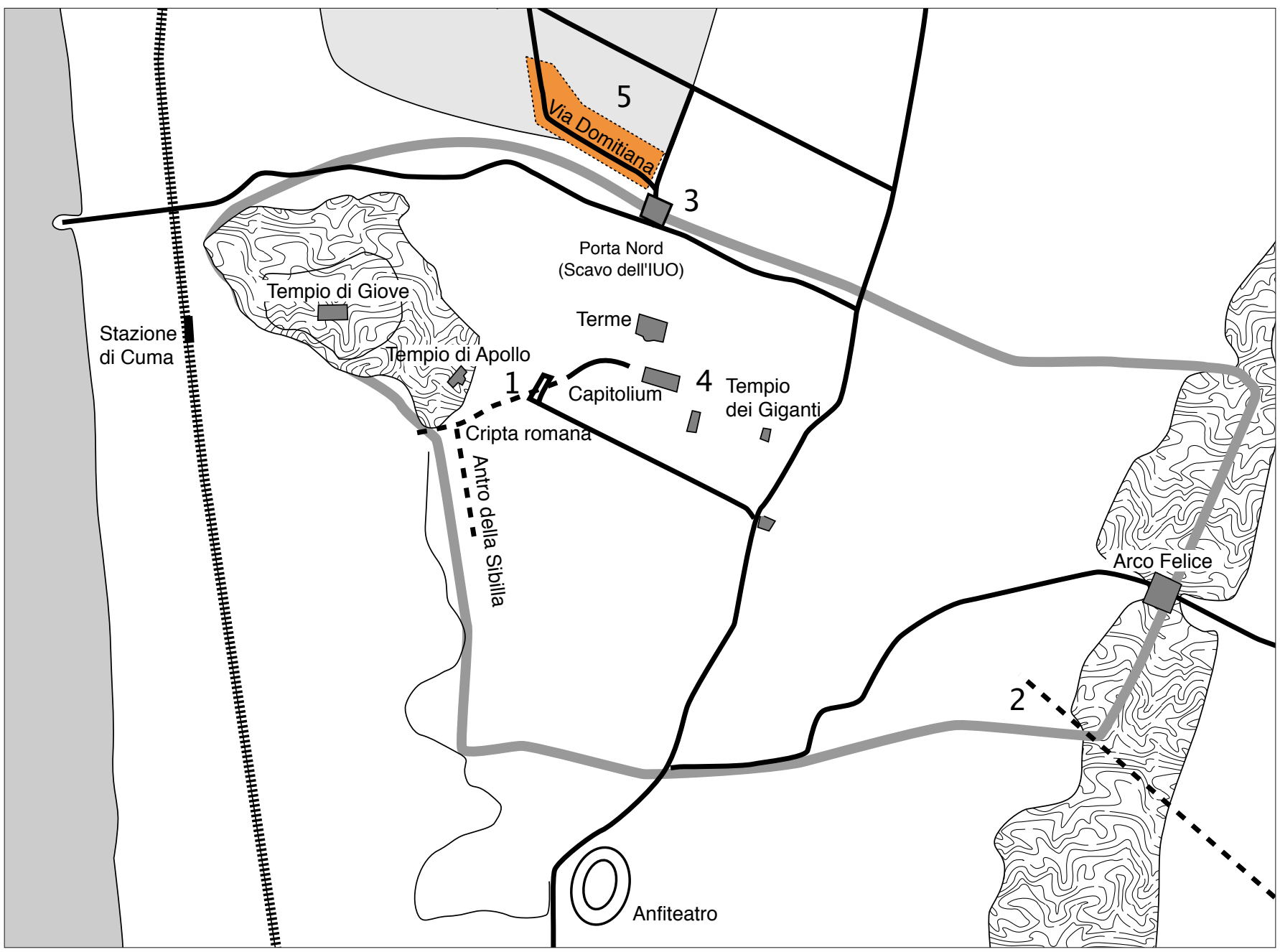

Fig. 2. Localisation des découvertes de creusets sur le site de Cumes (plan CJB) 1 : la via per la crypta romana ; 2 la Masseria Turrà ; 3 : la porte médiane ; 4 : le forum ; $5:$ le dépotoir hors les murs

\section{Cumes, centre producteur de bleu égyptien?}

Les recherches menées sur le site de Cumes ne cessent de montrer le rôle que la cité a pu jouer dans le domaine de l'artisanat. De nombreux rebuts de cuisson de plats en céramique commune, en vernis rouge pompéien ${ }^{11}$ et de gobelets en paroi fine ${ }^{12}$, sont des preuves, malgré l'absence de découverte de fours, d'une activité artisanale notable. Nous pouvons ajouter au panel des activités artisanales de la cité la fabrication du caeruleum.

\section{Histoire des recherches}

La première mention de présence de bleu égyptien sur le site de Cumes remonte à la fin du XIX ${ }^{\mathrm{e}}$ siècle. En 1874, H. de Fontenay publie un article dans lequel il compare les boules de bleu égyptien trouvées à Autun et au Mont Beuvray à celles appartenant au Musée de Sèvres ${ }^{13}$. La collection du Musée de Sèvres est constituée d'échantillons rapportés par le duc de Luynes des tombeaux de la vallée du

9 Giacchero 1974 , p. $218, n^{\circ} 34,84$ et 85.

10 Pline l'Ancien, Hist. Nat., XXXVI, 194, traduction de J. André et R. Bloch (1981).

11 Chiosi 1996.

12 Cavassa 2004b.

13 Fontenay 1874. 
1

Cuma-crypta C/733/1

T

C/733/1
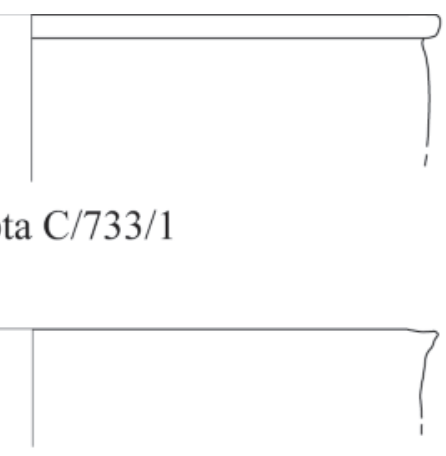

Cuma-crypta $\mathrm{C} / 733 / 3$
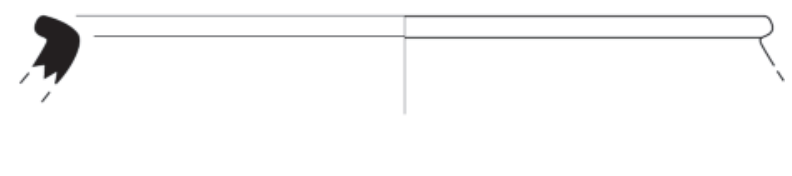

Cuma-crypta C/733/2

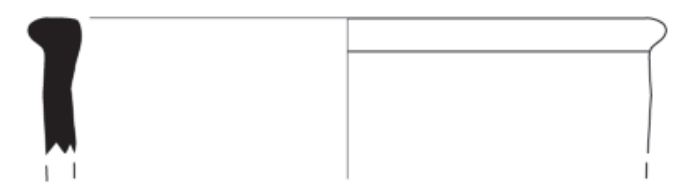

Cuma-crypta C/734/41

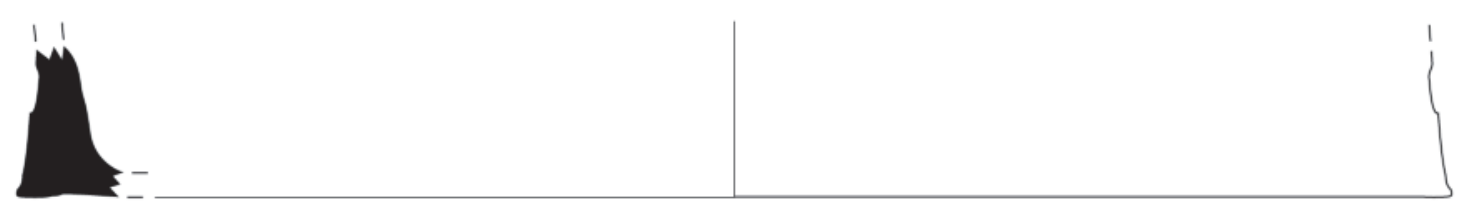

Cuma-crypta C/733/4

Echelle 1: 4

Fig. 3. Creusets des fouilles de la Via per la Crypta Romana (dessins L. C.)

Nil, des ruines de Thèbes, et ceux issus de la collection Passalacqa trouvés à Cumes et Pompéi.

Les recherches effectuées entre les $\mathrm{XIX}^{\mathrm{e}}$ et $\mathrm{XX}^{\mathrm{e}}$ siècles à Cumes et dans les Champs Phlégréens n'ont pas toujours été le fruit de fouilles programmées et systématiques. Une telle discontinuité fut causée en partie par les interruptions durant la Première et la Seconde Guerre Mondiale et par la difficile reprise des activités de recherche durant les années d'après guerre.

Les années 1980 marquent, suite aux phénomènes sismiques et de bradisisme que connut la Campanie, une rupture avec le passé et « l'avènement d'une ère nouvelle » pour la recherche archéologique à Cumes et dans les Champs Phlégréens. Plus particulièrement, à partir des années 1990, des interventions systématiques et régulières ont permis, grâce au développement des recherches, d'effectuer de nouvelles découvertes dans tous les domaines et dans la presque totalité des sites.
C'est le cas également du bleu égyptien pour lequel il faudra attendre, en fait, l'article de Patrizia Gargiulo en 1998 pour en voir réapparaître la mention à Cumes ${ }^{14}$. P. Gargiulo mentionne la présence d'échantillons de pigment bleu, mais également de nombreux fragments céramiques dont les parois sont recouvertes de ce même bleu ${ }^{15}$. Ces céramiques sont du même type que celles qu'elle a mises au jour sur le site de Liternum ${ }^{16}$. Il s'agit en fait de creusets ayant servi à la fabrication du bleu égyptien.

Les recherches de ces denières années ont mis au jour de nombreux fragments de creusets sur l'ensemble de la ville antique (fig. 2).

\footnotetext{
14 Gargiulo 1998, p. 61-65.

15 Gargiulo 1998, p. 64.

16 Gargiulo 1998.
} 

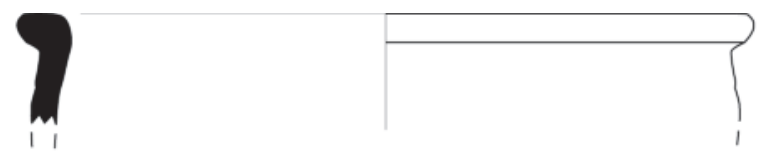

Cuma-Masseria Turrà 793/9

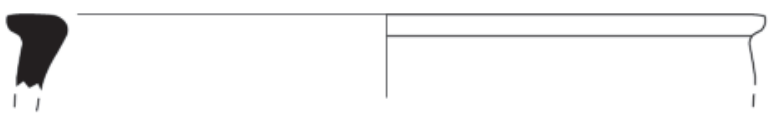

Cuma-Masseria Turrà 793/11
1

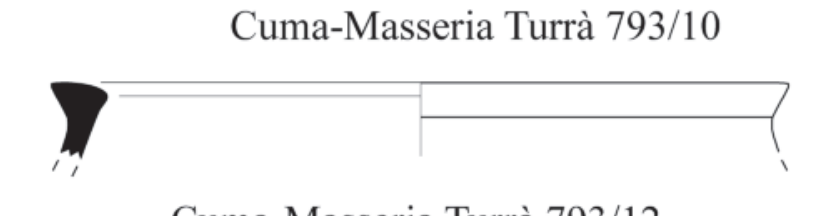

Cuma-Masseria Turrà 793/12

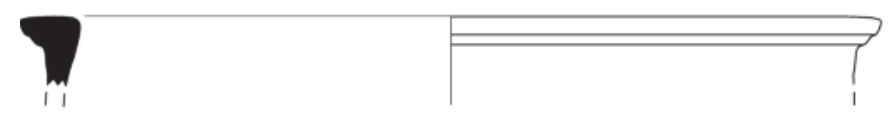

Cuma-Masseria Turrà 795/11
Echelle 1: 4

Fig. 4. Le matériel de la Masseria Turrà (dessins L.C.)

\section{Le matériel de la Via per la Crypta Romana)}

En 1992, à Cumes, durant un examen des lieux conduit par P. Caputo ${ }^{17}$, plusieurs fagments de ces creusets furent mis au jour dans la cité basse, sur le côté Nord de la voie dallée de blocs de basalte (fig. 2, 1), qui conduit du Forum vers la Crypta Romana (à l'emplacement de l'actuel petit pont de fer reliant deux parcelles d'un même terrain, actuellement acquis par la Surintendance dans le but d'agrandir le parc archéologique de la cité antique).

Une première étude de ces fragments (effectuée uniquement sur la base de la couleur, du traitement des parois internes et externes et du type, de la couleur et de l'épaisseur de la pâte) a permis d'identifier au moins quinze récipients du même type (fig. 3). Il s'agit de vases de forme cylindrique ayant une hauteur comprise entre 30 et $50 \mathrm{~cm}$, similaires, comme nous l'avons vu, aux fragments mis au jour en 1995 à Liternum dans un contexte daté du $\mathrm{I}^{\mathrm{er}}$ siècle de notre ère et que l'on peut identifier avec le type de l'exemplaire entier (haut de $50 \mathrm{~cm}$ ) mis au jour dans la nécropole du site. Dans cette nécropole, cas unique sur 90 sépultures, ce creuset fut réutilisé comme tombe pour une sépulture à enchytrismos. Plus particulièrement, deux fragments de fonds sont caractérisés par une incrustation (ou une croûte) de couleur bleue plus ou moins intense sur les parois internes et également sur les parois externes, due, comme nous le verrons, à la manipulation de hautes températures nécessaires à la fusion des boules de bleu égyptien.
Il faut souligner l'intérêt que présente ce contexte cumain, car la partie Nord de la via qui conduit à la Crypta Romana, correspond, en effet, à une ouverture caractérisée par la présence de marches permettant l'accès à un espace vraisemblablement souterrain (ou en tout cas situé sous le niveau de la voie en basalte), inséré dans un contexte urbain daté de l'époque tardo-républicaine / début de l'époque Augustéenne. Cette datation repose sur l'observation des techniques de construction employées. Seule la réalisation de fouilles permettrait de définir avec plus de précisions la destination exacte de ces structures. Quoi qu'il en soit, celles-ci devaient représenter l'extrémité Sud d'un vaste complexe, sans doute de destination civile, qui par ses dimensions et sa position pourrait être en rapport avec les autres structures souterraines sur lesquelles est construite l'ancienne Masseria G. Poerio, récemment acquise par la Surintendance Archéologique de Naples et de Caserte, comme le montre une étude de ces structures et des alentours ${ }^{18}$.

17 Avec la précieuse collaboration de Monsieur Cesare Giordano, Assistant technique et Restaurateur, que nous remercions pour son aide.

18 Cette étude a été réalisée dans le cadre d'une thèse de Laurea en architecture présentée en 1999 à l'Université degli Studi di Napoli " Federico II », par l'Architecte Cristina Ciotti, que nous remercions pour nous avoir fourni ces informations. 

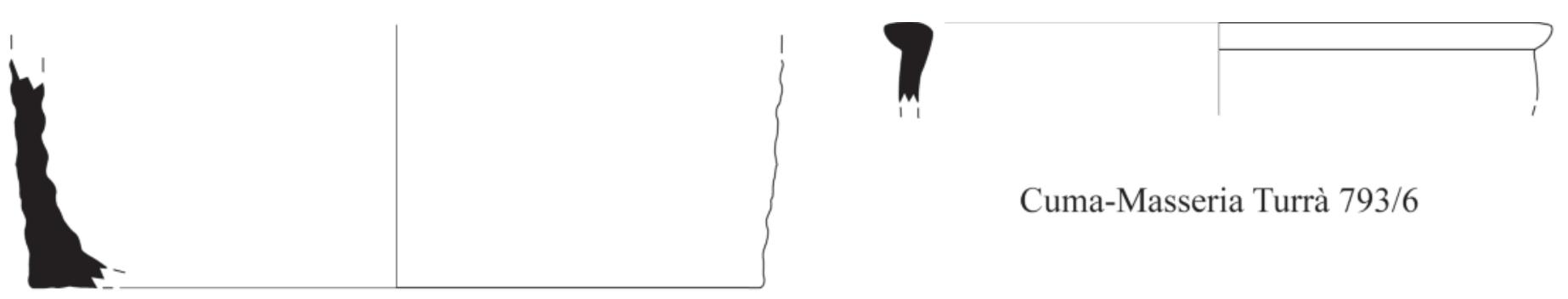

Cuma-Masseria Turrà 793/6

\section{Cuma-Masseria Turrà 786/1}
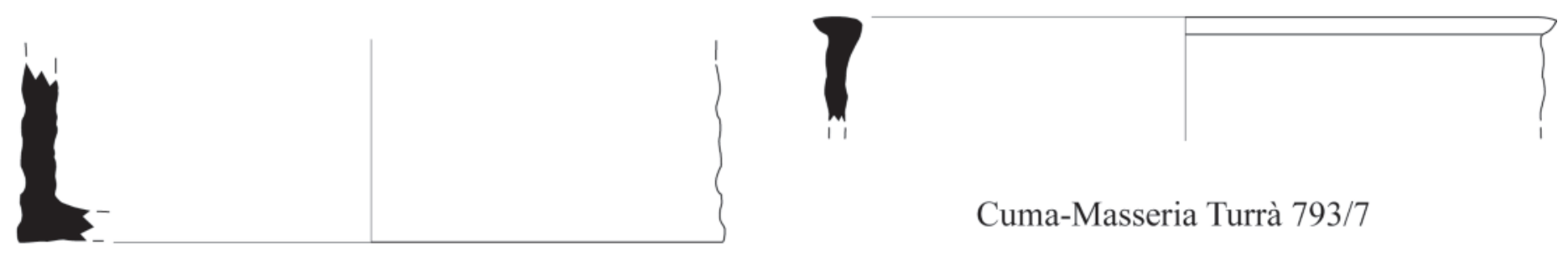

Cuma-Masseria Turrà 793/7

Cuma-Masseria Turrà 792/7

Echelle 1: 4

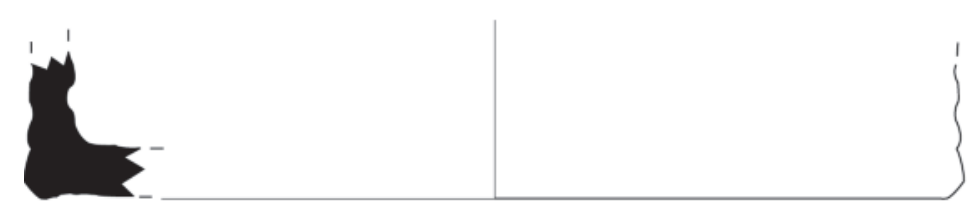

Cuma-Masseria Turrà 793/5

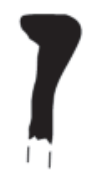

Cuma-Masseria Turrà /793/8

Fig. 5. Le matériel de la Masseria Turrà (dessins L.C.)

\section{La Masseria Turrà}

La masseria Turrà construite au XVIII ${ }^{\mathrm{e}}$ siècle, et successivement modifiée et agrandie, est située dans le secteur sud-est de la ville, sur des structures romaines, à proximité de la "Grotte de Cocceius " ${ }^{19}$. Dans l'aire environnante sont situés d'autres anciens vestiges dont la position sur un plateau (cote $39.8 \mathrm{~m}$ ) semble être particulièrement privilégiée par la vue qu'il offrait de la cité, du littoral et du port qu'abritait à l'époque le lac assaini de Licola. Les recherches archéologiques menées par $\mathrm{P}$. Caputo en 1997, ont permis d'identifier des structures relatives à une habitation du type domus dont les citernes sont utilisées comme basis villae, grâce à l'emplacement des éléments architecturaux et à la forme des terrasses (fig. 2, 2). L'ensemble est daté de la première moitié du $\mathrm{I}^{\text {er }}$ siècle avant J.-C. (pas au-

delà de 70) au I ${ }^{\text {er }}$ siècle après J.-C., bien que le site, pour divers motifs, ait été également fréquenté et modifié durant le Haut Moyen Âge (VI ${ }^{e}$ siècle après J.-C.). Les fouilles ont permis, entre autre, de mettre au jour 38 fragments de creusets (parmi lesquels 12 individus ont été dessinés : fig. 4 et 5) dans un contexte daté du ${ }^{\text {er }}$ siècle de notre ère ${ }^{20}$. Il s'agit de creusets cylindriques, hauts, dont le diamètre d'ouverture est d'environ $30 \mathrm{~cm}$. Une majorité de ces creusets présente des restes de pigment sur les parois (fig. 4

\footnotetext{
19 Caputo 2005, p. 39-42.

20 Caputo 2005, p. 42.
} 


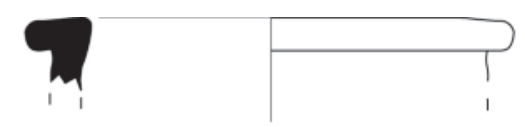

Cumes 300 027/8

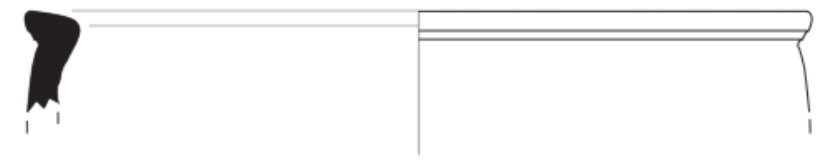

Cumes 300 022/9

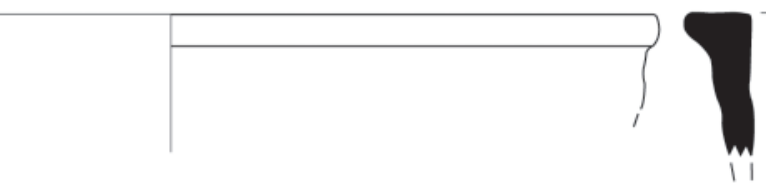

Cumes 100 050/10

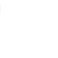

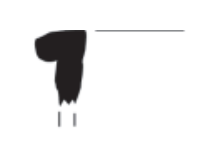

Cumes 5 001/11

problème de diamètre, fragment trop petit.
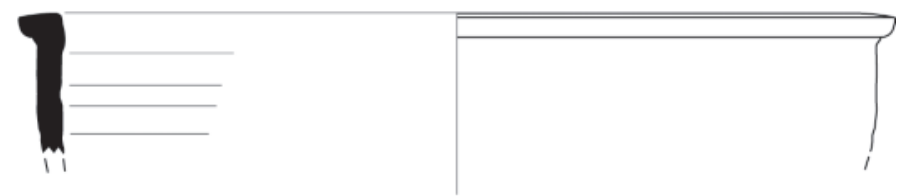

Cumes 300 011/12

\section{Cumes 11 005/13 Echelle 1:4}

Fig. 6. Le matériel du dépotoir d'époque Flavienne (dessins L.C.)

\section{et 5).}

La présence diffuse de ce type de matériel sur le site de Cumes, pour la première fois attesté dans des niveaux antiques, nous laisse hypothiser que dans la zone en amont de la masseria se trouvaient des officines et boutiques pour la fabrication du caeruleum. Si l'on excepte les restes épars, les murs de soutènement des terrasses, les restes d'anciennes carrières de tuf et les installations de "l'Arco Felice », la rare présence de structures antiques dans ce secteur de la ville correspondant à l'ample aire urbaine qui s'allonge parallèlement à la ligne de la colline de Montegrillo, en aval de " l'Arco Felice », semble indiquer que cette zone de la cité était sans doute faiblement urbanisée à l'époque et qu'elle était peutêtre destinée aussi à des activités artisanales.

\section{La porte médiane}

L'Università degli Studi "L'Orientale" di Napoli fouille depuis 1996 les murs septentrionaux de la cité. Son programme l'a amené à dégager et fouiller la porte dite "mediane » (fig. 2, 3). Lors de la fouille de ce secteur plusieurs fragments appartenants à ces mêmes creusets ont également été mis au jour. Les fragments sont décrits de la manière suivante : « (...) grosso contenitore in argilla grezza di colore giallo-verde, contenenti incrostazioni di una sostanza cristallina, di colore blu e azzurro, sulle pareti interne e in qualche caso di sostanza vetrificata sulle pareti esterne ${ }^{21}$. Une grande partie de ces fragments ont été mis au jour dans des dépôts datés du Haut Moyen Âge, mais contenant un abondan matériel résiduel daté du I $\mathrm{I}^{\mathrm{er}}$ - $\mathrm{II}^{\mathrm{e}}$ siècle de notre ère ${ }^{22}$. Ces dépôts correspondent à un rehaussement volontaire des niveaux de fréquentations dû à la montée du niveau de la lagune ${ }^{23}$.

\footnotetext{
21 Malpede 2003, p. 40.

22 Malpede 2003, p. 40.

23 Malpede 2005, p. 67.
} 


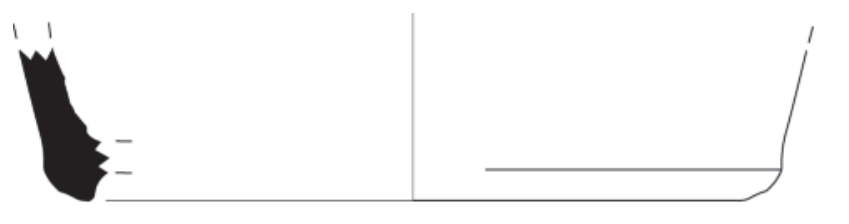

Cumes 300 027/19

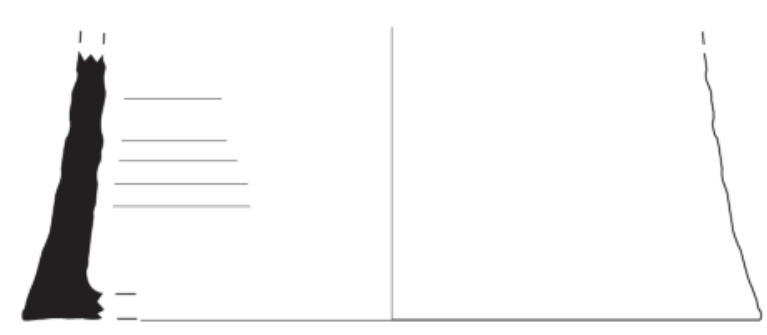

Cumes secteur $\mathrm{C} / 21$

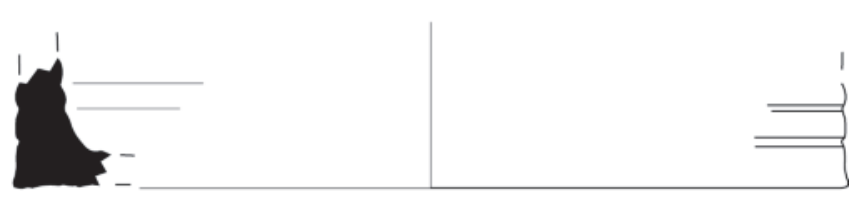

Cumes 11 005/20

Echelle $1: 4$

Fig. 7. Le matériel du dépotoir d'époque Flavienne (dessins L.C.)

\section{Le forum}

D'autres fragments céramiques avec des restes de caeruleum ont été mis au jour durant les fouilles du Capitolium et de la " Masseria del Gigante », dirigées par l'Université degli Studi di Napoli " Federico II » dans le cadre du projet « Kyme I » (1994-97), dans un contexte daté de l'époque tardo romaine $^{24}$ (fig. 2, 4). Puisqu'il s'agit de couches de remblayage et si l'on tient compte de la géomorphologie du site de Cumes et de l'emplacement du Forum, situé en aval de la Masseria Turrà, nous pouvons supposer que les fragments mis au jour sur le Forum auraient pu être emportés par les alluvions depuis l'aire située en amont. Cette zone correspond justement aux environs de la Masseria Turrà, où la présence d'officines est envisageable, vu sa position éloignée du centre de la ville.

\section{Les fouilles du Centre Jean Bérard}

Dans le cadre du projet "Kyme », le Centre Jean Bérard de Naples s'est chargé de la recherche des ports grecs et romains ainsi que des études géomorphologi- ques destinées à restituer le paysage du site ${ }^{25}$.

Parmi les zones fouillées, la principale se situe hors de la ville, au nord de la porte "médiane ». Cette fouille a permis de mettre au jour un tronçon de la voie Domitienne, bordée de nombreux monuments funéraires ${ }^{26}$, un puits et un égout, ainsi qu'un grand dépotoir flavien (fig. 2, 5). Dans ce dépotoir, de nombreux fragments de creusets ont été mis au jour ${ }^{27}$.

La fouille du dépotoir daté du début de la période flavienne a livré 153 fragments de creusets : 105 panses, 28 fonds, 20 bords, ainsi que de nombreux échantillons de pigment, sous forme de boulettes ou

24 Les informations nous ont été aimablement fournies par les Dottoresse C. Capaldi et F. Coraggio, que nous remercions. Caputo 2001, p. 49.

25 Projet dirigé par Jean-Pierre Brun et Priscilla Munzi.

26 Brun 2003, p. 131-155, Stefaniuk et alii, 2003, p. 399-435.

27 Ce travail a donné lieu à un sujet de DEA soutenu à l'Université d'Aix-en-Provence en 2004 sous la direction de M. Xavier Lafon. 

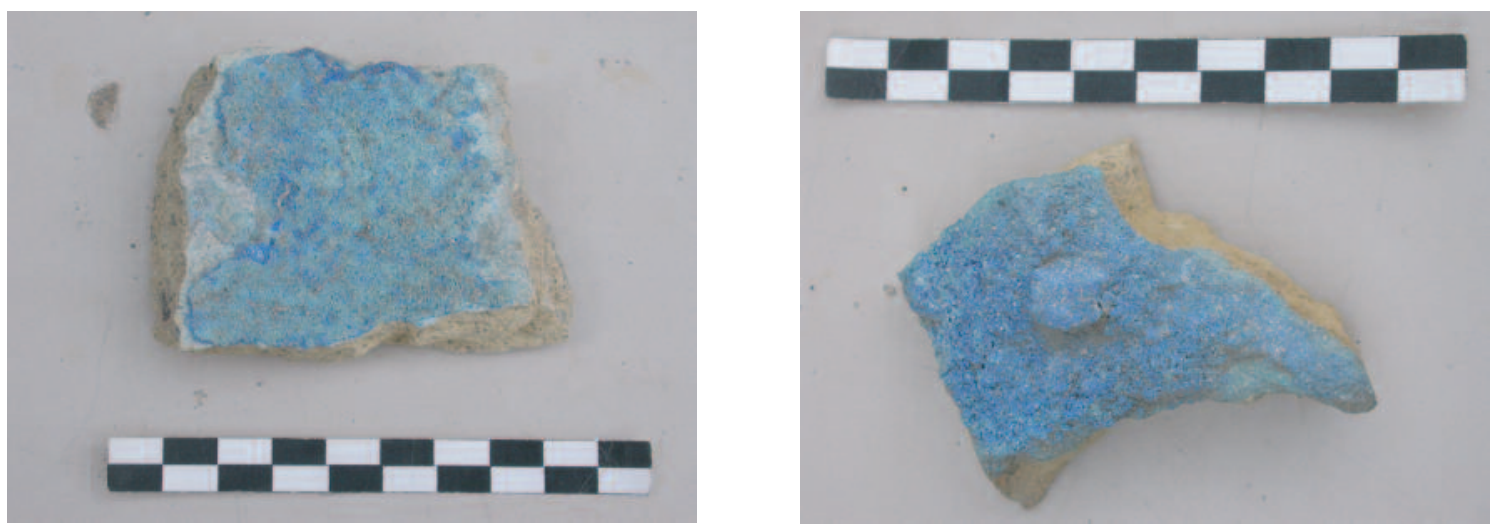

Fig. 8. Les creusets du dépotoir d'époque flavienne (cliché CJB)

de pains ${ }^{27}$. Il s'agit de vases de grandes dimensions, dont les parois sont épaisses d'environ $1 \mathrm{~cm}$ (fig. 67). La pâte renferme des inclusions grossières (argile, sable, cailloux...) ainsi que des particules volcaniques caractérisant une production locale. La grande majorité de ces fragments ont les parois recouvertes de bleu égyptien dont l'intensité de la couleur est plus ou moins forte. Certains ont également la particularité d'être déformés et recouverts de bleu sur les parois extérieures (fig. 8), comme c'est également le cas pour les fragments mis au jour dans les fouilles sur la via per la Crypta Romana : cela a très certainement été causé par les très fortes températures auquels les creusets et le pigment ont été exposés, et nous incite donc à y voir des traces de fabrication de bleu égyptien à Cumes.

Les recherches menées par D. Ullrich montrent la nécessité de porter le mélange à des températures avoisinant les $850-900^{\circ} \mathrm{C}$ pendant 24 à $48 \mathrm{~h}$. Il précise de plus l'importance de l'apport en oxygène lors de la fabrication du pigment : " thus production in an open crucible is not only possible but desirable. It is crucial to maintain a constant temperature over the entire production period, such as could be achieved by blowing air into the crucible ${ }^{28}$. La mention de "creusets ouverts" nécessaires lors de la fusion des éléments trouve son importance dans le cas des découvertes de Cumes car l'ensemble du matériel qu'il nous a été donné d'étudier rassemble des creusets dont le profil est celui d'un large pot dont les diamètres d'ouverture et de base oscillent autour des $30 \mathrm{~cm}$ et dont la hauteur est proche des 40-50 cm, en comparaison avec l'unique exemplaire intact mis au jour lors de la fouille de la nécropole du site de Liternum ${ }^{29}$.

\section{Conclusion}

Nous avons vu que ces creusets ont été mis au jour sur l'ensemble du territoire de la cité de Cumes (fig. 2). Les contextes de découverte permettent de faire les remarques suivantes :

- Les contextes datés montrent qu'au Ir siècle de notre ère, la cité de Cumes abritait un ou plusieurs ateliers de caeruleum. Le contexte le mieux daté, car clos, est le dépotoir d'époque flavienne situé hors de la ville. Nous pouvons supposer que la production se serait déplacée depuis Pouzzoles et/ou que des succursales auraient été ouvertes au $\mathrm{I}^{\text {er }}$ siècle de notre ère à Cumes et Liternum. Cette production reste-t-elle une spécialité de la gens Vestoria ? Change-t-elle de " mains » après un possible départ des descendants de Vestorius pour Pompéi ?

- Ces pots sont liés à la production et non au transport du produit. Un des exemples les plus parlants pour illustrer ce propos est la découverte de boules de bleu de Pouzzoles dans des épaves. Dans celle de Planier III ${ }^{30}$, les boulettes ont été retrouvées en vrac dans la cale du navire ; elles devaient être transportées dans des sacs et non dans des conteneurs céramiques qui eussent été inutilement lourds et encombrants.

- Les découvertes de creusets semblent concentrées dans les Champs Phlégréens. Peut-on y voir un monopole de la région pendant une certaine période au moins?

\footnotetext{
28 Ullrich 1987, p. 331.

29 Gargiulo 1998.

30 Tchernia 1970.
} 


\section{Bibliographie}

Andreau 1983 : ANDREAU (J.), À propos de la vie financière à Pouzzoles : Cluvius et Vestorius, in: Les bourgeoisies municipales italiennes aux $I I^{e}$ et $I^{e r}$ siècles avant J.-C., Centre Jean Bérard, Institut français de Naples, 7-10 décembre 1981, Paris, 1983, p. 9-20.

Brun 2003 : BRUn (J-P.) et alii, Alla ricerca del porto di Cuma. Relazione preliminare sugli scavi del Centre Jean Bérard, AION, Nuova Serie N. 7, Napoli, 2003, p. 131-155.

Caputo 2001 : CAPUTO (P.), Nuovi rinvenimenti vitrei dall'area archeologica di Cuma - Prospettive di ricerca, in : Piccioli (C.), Sogliani (F.) (a cura di), Il vetro in Italia Meridonale ed Insulare, VII Giornate Nazionali di Studio Comitato nazionale Italiano AIHV, Atti del Secondo Convegno Multidisciplinare, a cura di, Napoli 5-6-7 dicembre 2001, p. 45-51

Caputo 2005 : CAPUTO (P.), Una domus-villa urbana a Cuma, in Campania, e il suo rapporto con la città, Otium, Festschrift für Volker Michael Strocka, Sonderdruck, 2005, p. 39-46.

Cavassa 2004 : CAVASSA (L.), Production et diffusion du bleu de Pouzzoles, mémoire de DEA soutenu à l'Université de Provence, 2004.

Cavassa 2004a : Cavassa (L.), La vaisselle de Cumes (Italie) : deux études de cas ( $\mathrm{I}^{\text {er }}$ siècle avant J.-C. / I ${ }^{\text {er }}$ siècle après J.-C.), in : Actes du congrès de la SFECAG de Vallauris, 20-23 mai 2004, Marseille, 2004, p. 79-84.

Chiosi 1996 : Chiosi (E.), Cuma : una produzione di ceramica a vernice rossa interna, in : BATs (M.) (dir.), Les céramiques communes de Campanie et de Narbonnaise (I ${ }^{r}$ S. av. J.-C.-II ${ }^{e}$ s. ap. J.-C.), la vaisselle de cuisine et de table, Actes des Journées d'étude organisées par le Centre Jean Bérard et la Soprintendenza Archeologica per le Province di Napoli e Caserta, Naples 27-28 mai 1994. Naples, Collection du Centre Jean Bérard, 14, 1996, p. 225-233.

Delamare 2003 : Delamare (F.), La recette du caeruleum de Vitruve. Le point de vue de la science des matériaux, Archives Internationales d'Histoire des Sciences, 150-151, 53, 2003, p. 3-18.

Fontenay 1874 : Fontenay (H. de), Notes sur les couleurs antiques trouvées à Autun et au mont Beuvray, Mémoires de la Société Éduéenne, III, 1874, p. 451-477.

Forbes 1965 : Forbes (R. J.), Paints, pigments, inks and varnishes, chap. VII, Studies in ancient technology, volume III, Leiden, E. J. Brill, 1965, p. 210-257.

Gargiulo 1998 : GargiUlo (P.), Contenitori con depositi di colore blu egiziano e officine vetrarie nell'area dell'antica Liternum e nel territorio Flegreo. Aspetti tecnologici e prospettive di studio, in : Piccioli (C.), SOGLiani (F.) (a cura di), Il vetro dall'antichità all'età contemporanea : aspetti tecnologici, funzionali e commerciali, Atti $2^{e}$ giornate Nazionali di studio AIHVComitato Nazionale Italiano, 14-15 dicembre 1996, Milano, 1998, p. 61-65 et pl. V.

Giacchero 1974 : Giacchero (M.) (ed.), Edictum Diocletiani et Collegarum de pretiis rerum uenalium. Genova, 1974.

Lee 2000 : LeE (L.) et Quirke (S.), Painting materials, in : Nicholson P. T., ShaW (I.), Ancient Egyptian materials and technology, Cambridge University Press, 2000, p. 104-120.

Malpede 2003 : MALPEDE (V.), Cuma : reperti vitrei dalle recenti indagini dell'Istituto Universitario Orientale di Napoli nell'area delle fortificazioni settentrionali, in : Piccioli (C.) e Sogliani (F.), (a cura di), Il vetro in Italia Meridonale ed Insulare, VII Giornate Nazionali di Studio Comitato nazionale Italiano AIHV, Atti del Secondo Convegno Multidisciplinare, 5-6-7 dicembre 2001, Napoli, 2003, p. 33-43.

Malpede 2005 : MALPEDE (V.), Le fortificazioni settentrionali : l'area della porta "mediana ", in : D'Agostino (B.), Fratta (F.), Malpede (V.), Cuma. Le fortificazioni. 1. Lo scavo 1994-2002, Soprintendenza archeologica per le provincie di Napoli e Caserta, Università degli studi di Napoli "L'Orientale", dipartimento di studi del mondo classico e del Mediterraneo antico, AION, ArchStAnt. Quad. 15, Napoli, 2005, p. 23-77.

Martin 1985 : MARTIN (P. M.), La campanie antique des origines à l'éruption du vésuve. Peuplement et développement. Adosa, Clermont-ferrand, 1985.

Morel 1983 : MoRel (J.-P.), Les producteurs de biens artisanaux en Italie à la fin de la République, in : Les bourgeoisies municipales italiennes aux $I I^{e}$ et $I^{e r}$ siècles av. J.-C., Centre Jean Bérard, Institut français de Naples, 710 décembre 1981. Naples, 1983, p. 21-39.

Sirago 1979 : SiRAGO (V. A.), La personalità di C. Vestorio, Puteoli III, 1979, p. 3-16.

Stefaniuk et alii. 2003 : StefaniuK (L.), BRUn (J.-P.), Munzi (Pr.), MoRHANGe (Chr.), L'evoluzione dell'ambiente nei campi flegrei e le sue implicazioni storiche : il caso di Cuma e le ricerche del Centre Jean Bérard nella laguna di Licola, in : Convegno di studio sulla Magna Grecia, 42, Taranto, 2002, Napoli, 2003, p. 399-435.

Tchernia 1970 : TCHERnia (A.), Premiers résultats des fouilles de juin 1968 sur l'épave 3 de Planier, Études classiques 1968-1970, p. 51-82.

Ullrich 1987 : UllRich (D.), Egyptian Blue and Green frit : Characterization, history and occurrence, synthesis, in : Delamare (F.), Hackens (T.), Helly (B.) (éd.), Datation-Caractérisation des peintures pariétales et murales, PACT 17, Ravello, 1987, p. 323-332. 